\title{
Origin of superconductivity at nickel-bismuth interfaces
}

\author{
Matthew Vaughan $\odot,{ }^{1}$ Nathan Satchell $\odot,{ }^{1}$ Mannan Ali, ${ }^{1}$ Christian J. Kinane, ${ }^{2}$ \\ Gavin B. G. Stenning, ${ }^{2}$ Sean Langridge $\odot,^{2}$ and Gavin Burnell $\odot^{1, *}$ \\ ${ }^{1}$ School of Physics and Astronomy, University of Leeds, Leeds LS2 9JT, United Kingdom \\ ${ }^{2}$ ISIS Neutron and Muon Source, Rutherford Appleton Laboratory, Harwell, Oxon OX11 0QX, United Kingdom
}

(Received 22 November 2019; accepted 4 February 2020; published 6 March 2020)

\begin{abstract}
Unconventional superconductivity has been suggested to be present at the interface between bismuth and nickel in thin-film bilayers. In this work, we study the structural, magnetic, and superconducting properties of sputter deposited Bi/Ni bilayers. As-grown, our films do not display a superconducting transition; however, when stored at room temperature, after about 14 days our bilayers develop a superconducting transition up to $3.8 \mathrm{~K}$. To systematically study the effect of low temperature annealing on our bilayers, we perform structural characterization with $\mathrm{x}$-ray diffraction and polarized neutron reflectometry, along with magnetometry and low-temperature electrical transport measurements on samples annealed at $70^{\circ} \mathrm{C}$. We show that the onset of superconductivity in our samples is coincident with the formation of ordered $\mathrm{NiBi}_{3}$ intermetallic alloy, a known $s$-wave superconductor. We calculate that the annealing process has an activation energy of $(0.86 \pm 0.06) \mathrm{eV}$. As a consequence, gentle heating of the bilayers will cause formation of the superconducting $\mathrm{NiBi}_{3}$ at the $\mathrm{Ni} / \mathrm{Bi}$ interface, which poses a challenge to studying any distinct properties of $\mathrm{Bi} / \mathrm{Ni}$ bilayers without degrading that interface.
\end{abstract}

DOI: 10.1103/PhysRevResearch.2.013270

\section{INTRODUCTION}

Superconductivity and ferromagnetism are normally considered incompatible phases as the strong exchange field of a ferromagnet will act to break superconducting Cooper pairs [1]. It is therefore unusual to find a superconducting transition at about $4 \mathrm{~K}$ in $\mathrm{Bi} / \mathrm{Ni}$ bilayers, when ferromagnetic $\mathrm{Ni}$ has no known such transition, and crystalline $\mathrm{Bi}$ is only superconducting below $0.5 \mathrm{mK}$ [2]. Higher critical temperatures in $\mathrm{Bi}$ have been reported under certain conditions, for example, $T_{c} \approx 6 \mathrm{~K}$ in amorphous $\mathrm{Bi}, T_{c} \approx 4 \mathrm{~K}$ induced under pressures of a few $\mathrm{GPa}$, a $T_{c}$ range of between 2 and $5.5 \mathrm{~K}$ on the surface of grain boundaries and $T_{c}$ of $1.3 \mathrm{~K}$ in nanowires [3-7]. None of these can, however, explain the superconductivity in $\mathrm{Bi} / \mathrm{Ni}$ bilayers.

$\mathrm{Bi} / \mathrm{Ni}$ bilayer superconductivity was initially discovered in $\mathrm{Bi}$ layers grown on a dusting of $\mathrm{Ni}$ in tunneling measurements, which showed that the superconductivity extends across the entire thickness of the $\mathrm{Bi}$ [8]. Later, similar measurements showed that in such bilayers, superconductivity and ferromagnetism coexist [9]. Recently, there is a renewed interest in $\mathrm{Bi} / \mathrm{Ni}$ bilayers as the combination of superconductivity, ferromagnetism and strong spin-orbit coupling may lead to exotic new physics. In particular, epitaxial bilayers of $\mathrm{Bi} / \mathrm{Ni}$ grown by molecular beam epitaxy have been heavily studied

\footnotetext{
*g.burnell@leeds.ac.uk

Published by the American Physical Society under the terms of the Creative Commons Attribution 4.0 International license. Further distribution of this work must maintain attribution to the author(s) and the published article's title, journal citation, and DOI.
}

[10-16]. There is speculation that results on these epitaxial bilayers show $p$-wave superconductivity [10], time-reversal symmetry breaking [11], and chiral superconductivity [12].

An alternative explanation for the origin of the superconductivity in the $\mathrm{Bi} / \mathrm{Ni}$ bilayer is by the presence of the alloy $\mathrm{NiBi}_{3}$ which is established to superconduct with a similar $T_{c}$ of $4 \mathrm{~K}[17,18]$. Measurements on bulk crystals of $\mathrm{NiBi}_{3}$ suggest that it shows coexistence of superconductivity and ferromagnetism [19]; however, it is expected to be a singlet, $s$-wave, superconductor [20]. Silva et al. observed this alloy in thin-film Bi-Ni interfaces, which they attribute to spontaneous formation during sample growth at a temperature of $60^{\circ} \mathrm{C}$ [19]. Liu et al. also observe interdiffusion during sample growth at $300 \mathrm{~K}$ but no interdiffusion when samples are grown colder than $110 \mathrm{~K}$ [21]. Whilst formation during growth will be dominated by comparatively rapid surface diffusion, it is also important to establish whether, and under what conditions, formation of $\mathrm{NiBi}_{3}$ can occur post-growth when the as-grown samples show initially clean and distinct interfaces of $\mathrm{Bi} / \mathrm{Ni}$.

In this work, we set out to determine at what timescales and temperatures intermixing from an initially distinct $\mathrm{Bi} / \mathrm{Ni}$ interface becomes significant. Annealing at low temperatures, we use SQUID magnetometry, $x$-ray diffraction (XRD), and polarized neutron reflectometry (PNR) to measure changes in $\mathrm{Bi} / \mathrm{Ni}$ samples. X-ray reflectometry is not used as the $\mathrm{X}$-ray scattering density for bulk $\mathrm{Bi}$ and $\mathrm{Ni}$ are too similar for effective contrast at the interface. We observe the onset of a superconducting $T_{c}$, evolution of the magnetic moment, and changing structure of the interface. Our results suggest that special handling of the $\mathrm{Bi} / \mathrm{Ni}$ samples and refrigerated storage is necessary to prevent the formation of $\mathrm{NiBi}_{3}$, which otherwise occurs after a few days at room temperature, or 
a few minutes at temperatures typical of many cleanroom processing steps.

\section{METHODS}

Samples are grown by DC sputtering from pure metal targets of $\mathrm{Bi}(4 \mathrm{~N})$ and $\mathrm{Ni}(3 \mathrm{~N} 5)$. To provide the cleanest possible growth condition, a separate $\mathrm{Nb}$ target is used as a getter and a liquid nitrogen Meissner trap is used to further reduce the residual water. The base pressure of our system is $1.2 \times 10^{-5} \mathrm{~Pa}$ and substrate temperature is $21^{\circ} \mathrm{C}$. The substrate is thermally oxidized $\mathrm{Si}$ with $100 \mathrm{~nm}$ of $\mathrm{SiO}_{2}$ cleaned using $5 \mathrm{~min}$ of acetone and then isopropyl ultrasonic cleaning. Each target is presputtered for $5 \mathrm{~min}$. The growth pressure is $0.43 \mathrm{~Pa}$ for $\mathrm{Bi}$ and $0.61 \mathrm{~Pa}$ for $\mathrm{Ni}$ in a pure argon $(6 \mathrm{~N})$ atmosphere, the pressure distance product is $4.4 \mathrm{~Pa} \mathrm{~cm}$ and $3.8 \mathrm{~Pa} \mathrm{~cm}$, respectively. Growth rates are calibrated by fitting to Keissig fringes obtained by low-angle $\mathrm{x}$-ray reflectometry on single layer reference sample and are $4.3 \AA_{\mathrm{s}^{-1}}$ for $\mathrm{Bi}$ and $3.0 \AA \mathrm{s}^{-1}$ for Ni.

To prevent unintentional annealing, directly after removing from the deposition system, the samples are stored in a domestic freezer (Beko fridge/freezer model CDA543FW) at $\approx-20^{\circ} \mathrm{C}$ and transported in a portable refrigerator (Halfords $24112 \mathrm{~V}$ Electric Coolbox) at $\approx 4^{\circ} \mathrm{C}$. Samples were vacuum packed in airtight plastic to minimize condensation. Thirty-one days elapsed between sample growth and PNR measurements. We either anneal our samples at room temperature $\left(21^{\circ} \mathrm{C}\right)$ or perform controlled annealing of our samples between $50^{\circ} \mathrm{C}$ and $150^{\circ} \mathrm{C}$ on a hotplate under a cover to maintain a uniform temperature.

Polarized neutron reflectometry (PNR) is performed on the PolRef beamline at the ISIS neutron and muon source. Polarized neutron reflectometry data are analyzed using the GenX software [22]. X-ray diffraction (XRD) is performed on a Rigaku SmartLab diffractometer using $\mathrm{Cu} \mathrm{K}_{\alpha} \lambda=1.54 \AA$ radiation and magnetization loops are measured using a Quantum Design MPMS 3 SQUID magnetometer both courtesy of the ISIS R53 characterization laboratory. We also employ an Oxford Instruments MagLab 8 T VSM for additional magnetization measurements for the temperature dependent annealing. Electrical transport measurements are performed using a standard 4-point probe AC method utilizing a lock-in amplifier and a $77 \mathrm{~Hz}, 100 \mu \mathrm{A}$ current inside a ${ }^{4} \mathrm{He}$ variable temperature cryostat with a $3 \mathrm{~T}$ superconducting magnet in a horizontal Helmholtz Coil configuration.

\section{RESULTS}

\section{A. Optimization of the superconducting phase}

We first study the dependence of the superconducting $T_{c}$ on the thickness of the Bi and Ni layers in fully annealed samples (Fig. 1). Using the previous work [10] as a guide, we first fix the thickness of the Ni layer at $6 \mathrm{~nm}$ and vary the thickness of the Bi layer in the range $35-60 \mathrm{~nm}$ [Fig. 1(a)]. The $T_{c}$ is found initially to be $2.8 \mathrm{~K}$ for the thinnest $\mathrm{Bi}$, increasing to $3.7 \mathrm{~K}$ at $50 \mathrm{~nm}$, where the $T_{c}$ saturates and remains constant with further increasing Bi thickness. Next, we fix the thickness of the Bi layer at $50 \mathrm{~nm}$ and vary the thickness of the Ni layer in the range $4-10 \mathrm{~nm}$ [Fig. 1(b)]. Here, we see the highest $T_{c}$
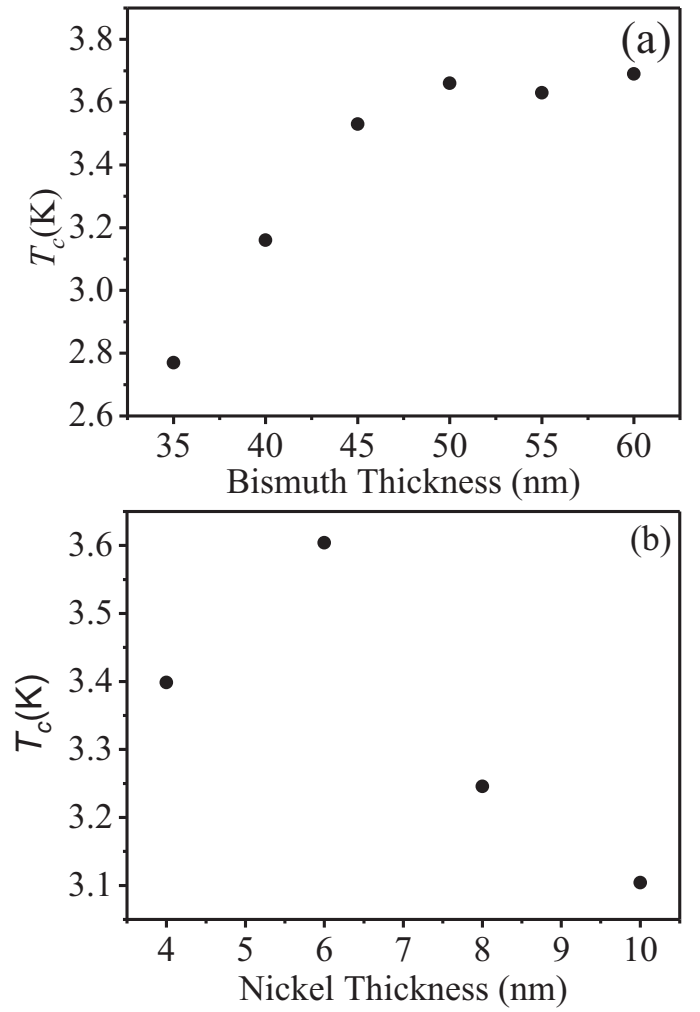

FIG. 1. Optimization of superconducting $T_{c}$. (a) Ni layer fixed at $6 \mathrm{~nm}$ with Bi layer varied, after 2 weeks at room temperature. (b) $\mathrm{Bi}$ layer fixed at $50 \mathrm{~nm}$ and $\mathrm{Ni}$ layer varied, annealed at $100^{\circ} \mathrm{C}$.

for $6 \mathrm{~nm}$ of $\mathrm{Ni}$, with lower $T_{c}$ when the thickness of the $\mathrm{Ni}$ is either reduced or increased from this value. The optimal layer thicknesses for maximum $T_{c}$ are, therefore, $50 \mathrm{~nm}$ of $\mathrm{Bi}$ and $6 \mathrm{~nm}$ of Ni, consistent with previous work [10].

\section{B. Polarized neutron reflectometry measurements}

By measuring the neutron reflectivity as a function of the wave-vector transfer and neutron spin eigenstate, PNR allows the scattering length density (SLD) to be obtained. Careful fitting to the two obtained reflectivity curves in PNR allows the extraction of depth dependent magnetization and structure. PNR is widely employed in the successful characterization of spintronic materials [23] and is particularly useful in this study due to the large SLD contrast between the Ni and Bi layers. The sample is annealed repeatedly at $70^{\circ} \mathrm{C}$. Films are loaded at room temperature and cooled in an $0.2 \mathrm{~T}$ field to $10 \mathrm{~K}$ where we perform PNR measurements.

Figures 2(a) and 2(c) show the obtained PNR curves for the sample with the corresponding fit to each spin state. Figure 2(a) shows the as-grown state, while Fig. 2(c) is after $3600 \mathrm{~s}$ annealing at $70^{\circ} \mathrm{C}$. The higher frequency oscillations arise from the thick $\mathrm{SiO}_{2}$ layer on the substrate. The thinner, rougher, layers of the film modulate these oscillations. The spin asymmetry as calculated by $\frac{u-d}{u+d}$ is shown in Figs. 2(b) and 2(d). The spin asymmetry directly scales with the sample's magnetization. The spin asymmetry shows significant reduction between the as-grown and annealed states indicating a reduction of magnetization. 

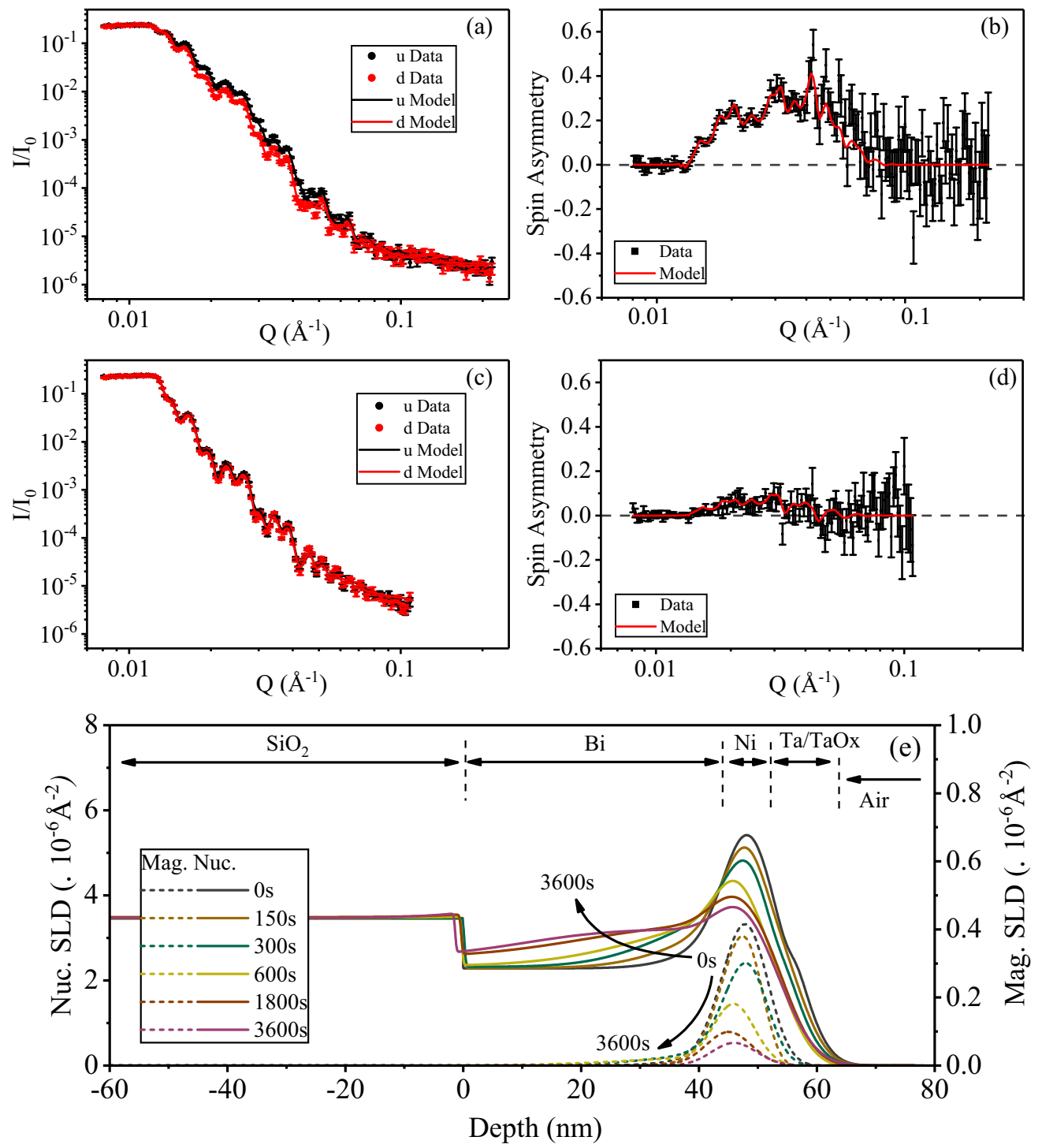

FIG. 2. Polarized neutron reflectometry (PNR) measurements at $10 \mathrm{~K}$ and $0.2 \mathrm{~T}$ of $\mathrm{Si} / \mathrm{SiO}(100 \mathrm{~nm}) / \mathrm{Bi}(50 \mathrm{~nm}) / \mathrm{Ni}(6 \mathrm{~nm}) / \mathrm{Ta}(5 \mathrm{~nm})$ sample. (a), (b) PNR and spin asymmetry in the as-grown state. (c), (d) PNR and spin asymmetry after $3600 \mathrm{~s}$ annealing at $70^{\circ} \mathrm{C}$. The solid lines are fits to the data and the returned fit parameters are given in Table I. (e) The nuclear and magnetic scattering length density (SLD) with depth returned from fitting showing the evolution of SLD with successive annealing.

We employ a box model to fit the PNR data with the layers $\mathrm{Ta}_{2} \mathrm{O}_{5} / \mathrm{Ta} / \mathrm{Ni} / \mathrm{NiBi}_{3} / \mathrm{Bi} / \mathrm{SiO}_{2} / \mathrm{Si}$. $\mathrm{Ta}_{2} \mathrm{O}_{5}$ is the most common oxide of $\mathrm{Ta}$ and the $\mathrm{SiO}_{2}$ density is taken as amorphous. Each layer is assigned a thickness, roughness, magnetization, scattering length, and density. The scattering lengths are fixed from the known bulk values. Magnetism in the structure is limited to the $\mathrm{Ni}$ and $\mathrm{NiBi}_{3}$ layers, $\mathrm{NiBi}_{3}$ is not ferromagnetic, and we do not find a significant moment inside this layer. All other parameters except the substrate density and thickness (the substrate is infinitely thick) are free fitting parameters with physically realistic bounds defined from either the known bulk or as-grown values. The results of fitting are shown in Fig. 2(e) and Table I.

The results of fitting are shown in Fig. 2(e). As grown the samples do show a thin $(2 \mathrm{~nm}) \mathrm{NiBi}_{3}$ layer that has either formed during growth, or in the time taken to remove them from the deposition system. Consequently, the $\mathrm{Ni}$ and $\mathrm{Bi}$ layers are slightly thinner than expected (4.4 and $44 \mathrm{~nm}$ versus the nominal 6 and $50 \mathrm{~nm}$ ). The thickness of the $\mathrm{NiBi}_{3}$ layer in the model is less than the roughness of any of the $\mathrm{Bi}, \mathrm{NiBi}_{3}$, or $\mathrm{Ni}$ interfaces suggesting that it is an intermixed region rather than a distinct layer. The Magnetic SLD of bulk Ni is $\approx 1 \times$ $10^{-6} \AA^{-2}$ where our magnetic SLD for the Ni in the as-grown state is $\approx 0.4 \times 10^{-6} \AA^{-2}$ as the roughness distributes the $\mathrm{Ni}$ over a wider range. The area under the Magnetic SLD and the area derived from the box model with no roughness is $3.98 \times 10^{-6} \AA^{-1}$ and $3.97 \times 10^{-6} \AA^{-1}$, respectively.

The main changes to the structure with annealing the sample are the diffusion of $\mathrm{Ni}$ atoms into the $\mathrm{Bi}$ layer. The stronger nuclear scattering of the Ni increases the SLD of the Bi layer where atoms have diffused [Fig. 2(e)]. The structural profile of the final annealed state suggests that the $\mathrm{Ni}$ can diffuse across the entire thickness of the Bi as the SLD of the entire layer is raised. At the $\mathrm{Bi} / \mathrm{Ni}$ interface, we report that a layer with the correct SLD for ordered $\mathrm{NiBi}_{3}$ intermetallic layer is found. The thickness of this layer increases with 
TABLE I. Fitting parameters to the PNR data for each $70^{\circ} \mathrm{C}$ annealing step with the returned figure of merit (FOM). The Si substrate density is fixed to the bulk value and all other parameters are free fitting. The density for each free layer is fitted with tight bounds to remain physical. The initial and final layer thicknesses appear in bold to guide the reader. ${ }^{*}$ The final two Bi densities are higher that bulk as there is $\mathrm{Ni}$ inclusion throughout the layer. ${ }^{\dagger}$ GenX does not correctly fit very thin layers with large roughness.

\begin{tabular}{|c|c|c|c|c|c|c|}
\hline Anneal time: & $\begin{array}{c}0 \mathrm{~s} \\
\text { (initial) }\end{array}$ & $150 \mathrm{~s}$ & $300 \mathrm{~s}$ & $600 \mathrm{~s}$ & $1800 \mathrm{~s}$ & $\begin{array}{l}3600 \mathrm{~s} \\
\text { (final) }\end{array}$ \\
\hline 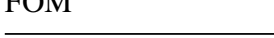 & & 1.341 & 1.415 & 1.404 & & \\
\hline $\begin{array}{l}\mathrm{Ta}_{2} \mathrm{O}_{5}: d(\mathrm{~nm}) \\
\rho(\% \text { bulk }) \\
\sigma(\mathrm{nm})\end{array}$ & $\begin{array}{l}\mathbf{1 . 6} \pm \mathbf{0 . 3} \\
95 \pm 6 \\
3.8 \pm 0.2\end{array}$ & $\begin{array}{l}1.2 \pm 0.2 \\
102 \pm 5 \\
4.0 \pm 0.1\end{array}$ & $\begin{array}{l}3.9 \pm 0.8 \\
85 \pm 9 \\
4.4 \pm 0.3\end{array}$ & $\begin{array}{l}2.4 \pm 0.5 \\
89 \pm 5 \\
4.2 \pm 0.3\end{array}$ & $\begin{array}{l}5.7 \pm 0.8 \\
83 \pm 3 \\
4.4 \pm 0.4\end{array}$ & $\begin{array}{l}\mathbf{4 . 0} \pm \mathbf{0 . 5} \\
118 \pm 8 \\
5.0 \pm 0.2\end{array}$ \\
\hline $\begin{array}{l}\text { Ta: } d(\mathrm{~nm}) \\
\rho(\% \text { bulk }) \\
\sigma(\mathrm{nm})\end{array}$ & $\begin{array}{l}\mathbf{4 . 9} \pm \mathbf{0 . 4} \\
92 \pm 1 \\
18 \pm 0.3\end{array}$ & $\begin{array}{l}5.1 \pm 0.7 \\
104 \pm 3 \\
4.2 \pm 0.6\end{array}$ & $\begin{array}{l}3.9 \pm 0.6 \\
103 \pm 6 \\
3.8 \pm 0.1\end{array}$ & $\begin{array}{l}3.3 \pm 0.3 \\
97 \pm 5 \\
1.5 \pm 0.9\end{array}$ & $\begin{array}{l}3.5 \pm 0.5 \\
103 \pm 5 \\
4.1 \pm 0.3\end{array}$ & $\begin{array}{l}\mathbf{0 . 5} \pm \mathbf{0 . 5} \\
80 \pm 10 \\
2.3 \pm 0.4\end{array}$ \\
\hline $\begin{array}{l}\mathrm{Ni}: d(\mathrm{~nm}) \\
\rho(\% \text { bulk }) \\
\sigma(\mathrm{nm}) \\
\mathrm{M}\left(\mu_{B} / \text { atom }\right)\end{array}$ & $\begin{array}{l}\mathbf{4 . 5} \pm \mathbf{0 . 7} \\
91 \pm 1 \\
3.2 \pm 0.2 \\
0.40 \pm 0.02\end{array}$ & $\begin{array}{l}3.5 \pm 0.1 \\
83 \pm 2 \\
2.7 \pm 0.2 \\
0.46 \pm 0.03\end{array}$ & $\begin{array}{l}2.4 \pm 0.1 \\
90 \pm 1 \\
3.9 \pm 0.2 \\
0.51 \pm 0.03\end{array}$ & $\begin{array}{l}2.1 \pm 0.1 \\
101 \pm 3 \\
3.3 \pm 0.2 \\
0.45 \pm 0.03\end{array}$ & $\begin{array}{l}0.7 \pm 0.2^{\dagger} \\
90 \pm 20 \\
3.9 \pm 0.2 \\
0.57 \pm 0.04\end{array}$ & $\begin{array}{l}\mathbf{0 . 7} \pm 0.1^{\dagger} \\
87 \pm 8 \\
4.0 \pm 0.2 \\
0.27 \pm 0.09\end{array}$ \\
\hline $\begin{array}{l}\mathrm{NiBi}_{3}: d(\mathrm{~nm}) \\
\rho(\% \text { bulk }) \\
\sigma(\mathrm{nm}) \\
\mathrm{M}\left(\mu_{B} / \text { atom }\right)\end{array}$ & $\begin{array}{l}\mathbf{2 . 3} \pm \mathbf{0 . 4} \\
92 \pm 2 \\
4.7 \pm 0.3 \\
0.03 \pm 0.1\end{array}$ & $\begin{array}{l}8.7 \pm 0.7 \\
103 \pm 3 \\
4.5 \pm 0.2 \\
0.01 \pm 0.05\end{array}$ & $\begin{array}{l}15 \pm 2 \\
99 \pm 1 \\
3.5 \pm 0.3 \\
0.01 \pm 0.02\end{array}$ & $\begin{array}{l}19 \pm 2 \\
96 \pm 7 \\
4.6 \pm 0.3 \\
0.01 \pm 0.02\end{array}$ & $\begin{array}{l}24 \pm 1 \\
99 \pm 2 \\
3.8 \pm 0.3 \\
0 \pm 0.03\end{array}$ & $\begin{array}{l}\mathbf{3 8 . 8} \pm \mathbf{0 . 7} \\
91 \pm 1 \\
5.2 \pm 0.3 \\
0 \pm 0.01\end{array}$ \\
\hline $\begin{array}{l}\mathrm{Bi}: d(\mathrm{~nm}) \\
\rho(\% \text { bulk }) \\
\sigma(\mathrm{nm})\end{array}$ & $\begin{array}{l}\mathbf{4 4 . 1} \pm \mathbf{0 . 4} \\
96 \pm 1 \\
7.9 \pm 0.9\end{array}$ & $\begin{array}{l}37.8 \pm 0.8 \\
95 \pm 2 \\
9.5 \pm 0.7\end{array}$ & $\begin{array}{l}30 \pm 1 \\
97 \pm 2 \\
7.9 \pm 0.8\end{array}$ & $\begin{array}{l}29 \pm 2 \\
97 \pm 1 \\
7 \pm 2\end{array}$ & $\begin{array}{l}20.9 \pm 0.8 \\
106 \pm 3^{*} \\
15 \pm 3\end{array}$ & $\begin{array}{l}\mathbf{9 . 3} \pm \mathbf{0 . 7} \\
105 \pm 1^{*} \\
9 \pm 1\end{array}$ \\
\hline $\begin{array}{l}\mathrm{SiO}_{2}: d(\mathrm{~nm}) \\
\rho(\% \text { bulk }) \\
\sigma(\mathrm{nm})\end{array}$ & $\begin{array}{l}\mathbf{9 5 . 0} \pm \mathbf{0 . 3} \\
100.5 \pm 0.1 \\
0.1 \pm 0.4\end{array}$ & $\begin{array}{l}95.1 \pm 0.6 \\
99.8 \pm 0.2 \\
0.1 \pm 0.7\end{array}$ & $\begin{array}{l}95.1 \pm 0.3 \\
99.9 \pm 0.7 \\
0.2 \pm 0.5\end{array}$ & $\begin{array}{l}95.1 \pm 0.3 \\
99.9 \pm 0.3 \\
0.4 \pm 0.6\end{array}$ & $\begin{array}{l}94.7 \pm 0.4 \\
100.6 \pm 0.3 \\
0.2 \pm 0.4\end{array}$ & $\begin{array}{l}\mathbf{9 3 . 9} \pm \mathbf{0 . 5} \\
100.1 \pm 0.2 \\
0.2 \pm 0.6\end{array}$ \\
\hline $\begin{array}{l}\text { Si: } \rho \text { Fixed }(\% \text { bulk }) \\
\sigma(\mathrm{nm})\end{array}$ & $\begin{array}{l}100 \\
0.1 \pm 0.3\end{array}$ & $\begin{array}{l}100 \\
0.1 \pm 0.4\end{array}$ & $\begin{array}{l}100 \\
0.2 \pm 0.3\end{array}$ & $\begin{array}{l}100 \\
0.2 \pm 0.3\end{array}$ & $\begin{array}{l}100 \\
0.2 \pm 0.2\end{array}$ & $\begin{array}{l}100 \\
0.3 \pm 0.2\end{array}$ \\
\hline
\end{tabular}

annealing from $(2 \mathrm{~nm})$ in the unannealed state, to $(38.8 \mathrm{~nm})$ in the final annealed state.

As the Ni diffuses; less ferromagnetically ordered Ni is left, in turn decreasing the magnetic SLD profile and moment measured independently from SQUID. For the final two annealing steps, the fitting returns a very thin layer of $\mathrm{Ni}(0.7 \mathrm{~nm})$. The large roughness $(4 \mathrm{~nm})$ of this layer suggests it is no longer continuous and has been replaced by an intermixing layer. In an attempt to improve the modeling, we try replacing this $\mathrm{Ni}$ layer with a layer approximating a NiBi intermixing layer for the final two annealing steps (Table II). Doing so, we find the fitting returns a lower figure of merit, indicating that model has a closer resemblance to the physical sample. This suggests that in the final annealed state, Bi contaminates the Ni layer in addition to the $\mathrm{Ni}$ diffusion into the $\mathrm{Bi}$ layer. There is very little change to the scattering length density shown Fig. 2(e) between the two models as the roughness tends to smear such fine details.

\section{Magnetometry and $x$-ray diffraction}

To study the magnetic and structural properties of our $\mathrm{Bi} / \mathrm{Ni}$ bilayer samples we employ SQUID magnetometry and $\mathrm{Cu} \mathrm{K} \mathrm{K}_{\alpha} \lambda=1.54 \AA \mathrm{x}$-ray diffraction (XRD). We use a sample grown in the same vacuum cycle as the sample we study by PNR, which we dice into smaller $(4 \times 4 \mathrm{~mm})$ cuttings. The individual cuttings are treated with the same annealing process at $70^{\circ} \mathrm{C}$ as the PNR sample. After annealing, we first measure the magnetic hysteresis (moment versus field) of the cuttings at $10 \mathrm{~K}$ (the same temperature as the PNR), then check for superconducting transition by measuring moment versus temperature from the base temperature of the magnetometer at small applied field (2-10 K sweep at $5 \mathrm{mT})$, finally we transfer the cuttings to the $\mathrm{X}$-ray diffractometer where XRD is measured at room temperature. To minimize annealing during the X-ray measurements, the total time to align and record a XRD scan is optimized to take about $30 \mathrm{~min}$. Nevertheless, we find by re-measuring a cutting's magnetic response after the room temperature XRD scan has finished, that some annealing occurs during the X-ray measurement. We do not believe this to have an influence on the results presented in this section, however as a precaution do not measure the same cutting more than once and each annealing time for SQUID and XRD measurements are from a different cutting of the pristine sample.

The magnetic characterization of our Bi/Ni bilayer samples are shown in Fig. 3. We extract the saturation (applied field of $1 \mathrm{~T})$ moment/area by measuring hysteresis loops for cuttings with different annealing times. Exemplar hysteresis loops are shown in the inset of Fig. 3. For the as-grown sample cutting, the saturation magnetization of the $4.4 \mathrm{~nm} \mathrm{Ni}$ layer is $318 \mathrm{emu} / \mathrm{cm}^{3}$, reduced from the bulk value of $600 \mathrm{emu} / \mathrm{cm}^{3}$. The reduced magnetization is consistent with the formation of magnetic dead layers, which are often observed in thin film 
TABLE II. Fitted parameters to the PNR data, with the returned figure of merit (FOM), when replacing the $\mathrm{Ni}$ layer with a $\mathrm{NiBi}$ intermixing layer for the final two annealing steps (see text).

\begin{tabular}{|c|c|c|}
\hline $\begin{array}{l}\text { Anneal time: } \\
\text { FOM }\end{array}$ & $\begin{array}{l}1800 \mathrm{~s} \\
1.167\end{array}$ & $\begin{array}{l}3600 \mathrm{~s} \\
\text { (final) } \\
1.807\end{array}$ \\
\hline $\begin{array}{l}\mathrm{Ta}_{2} \mathrm{O}_{5}: d(\mathrm{~nm}) \\
\rho(\% \text { bulk }) \\
\sigma(\mathrm{nm})\end{array}$ & $\begin{array}{l}2.2 \pm 0.4 \\
90 \pm 10 \\
4.4 \pm 0.2\end{array}$ & $\begin{array}{l}5.1 \pm 0.8 \\
79 \pm 2 \\
4.2 \pm 0.1\end{array}$ \\
\hline $\begin{array}{l}\text { Ta: } d(\mathrm{~nm}) \\
\rho(\% \text { bulk }) \\
\sigma(\mathrm{nm})\end{array}$ & $\begin{array}{l}2.2 \pm 0.4 \\
101 \pm 7 \\
4 \pm 2\end{array}$ & $\begin{array}{l}3.1 \pm 0.5 \\
103 \pm 2 \\
1 \pm 2\end{array}$ \\
\hline $\begin{array}{l}\text { NiBi: } d(\mathrm{~nm}) \\
\rho(\% \text { bulk }) \\
\sigma(\mathrm{nm}) \\
\mathrm{M}\left(\mu_{B} / \text { atom }\right)\end{array}$ & $\begin{array}{l}3.6 \pm 0.8 \\
108 \pm 9 \\
4 \pm 2 \\
0.4 \pm 0.1\end{array}$ & $\begin{array}{l}3.5 \pm 0.8 \\
90 \pm 3 \\
10 \pm 1 \\
0.5 \pm 0.2\end{array}$ \\
\hline $\begin{array}{l}\mathrm{NiBi}_{3}: d(\mathrm{~nm}) \\
\rho(\% \text { bulk }) \\
\sigma(\mathrm{nm}) \\
\mathrm{M}\left(\mu_{B} / \text { atom }\right)\end{array}$ & $\begin{array}{l}24 \pm 1 \\
98 \pm 2 \\
5.5 \pm 0.6 \\
0.02 \pm 0.02\end{array}$ & $\begin{array}{l}38 \pm 1 \\
88.4 \pm 0.8 \\
8.5 \pm 0.6 \\
0.00 \pm 0.03\end{array}$ \\
\hline $\begin{array}{l}\text { Bi: } d(\mathrm{~nm}) \\
\rho(\% \text { bulk }) \\
\sigma(\mathrm{nm})\end{array}$ & $\begin{array}{l}22 \pm 1 \\
106 \pm 2 \\
16 \pm 1\end{array}$ & $\begin{array}{l}4.5 \pm 0.4 \\
92 \pm 2 \\
3.6 \pm 0.5\end{array}$ \\
\hline $\begin{array}{l}\mathrm{SiO}_{2}: d(\mathrm{~nm}) \\
\rho(\% \text { bulk }) \\
\sigma(\mathrm{nm})\end{array}$ & $\begin{array}{l}94.4 \pm 0.3 \\
100.7 \pm 0.2 \\
0.1 \pm 0.5\end{array}$ & $\begin{array}{l}94.9 \pm 0.2 \\
100.0 \pm 0.3 \\
0.1 \pm 0.4\end{array}$ \\
\hline $\begin{array}{l}\text { Si: } \rho \text { Fixed }(\% \text { bulk }) \\
\sigma(\mathrm{nm})\end{array}$ & $\begin{array}{l}100.0 \\
0.1 \pm 0.3\end{array}$ & $\begin{array}{l}100.0 \\
0.1 \pm 0.3\end{array}$ \\
\hline
\end{tabular}

Ni [24]. For all sample cuttings, we find that a large magnetic field (nearly $1 \mathrm{~T}$ ) is required to achieve full saturation, hence the hysteresis loops shown in Fig. 3 inset do not fully close within the field range shown. As the film is annealed,

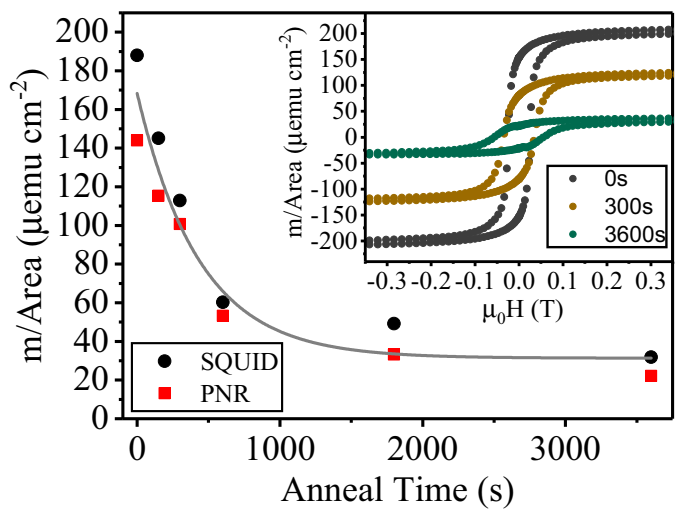

FIG. 3. Magnetic characterization at $10 \mathrm{~K}$ of $\mathrm{Si} / \mathrm{SiO}(100 \mathrm{~nm}) / \mathrm{Bi}$ $(50 \mathrm{~nm}) / \mathrm{Ni}(6 \mathrm{~nm}) / \mathrm{Ta}(5 \mathrm{~nm})$ samples. The moment per area calculated from the hysteresis loops, inset, are shown with annealing time at $70^{\circ} \mathrm{C}$. The diamagnetic contribution due to the substrate has been subtracted. The uncertainty in moment/area is dominated by the area measurements (different cuttings of the sample are used for each annealing step) and is less than 5\%. Also shown is the moment/area extracted by fitting the PNR measurements. The line on the main figure is a guide for the eye.
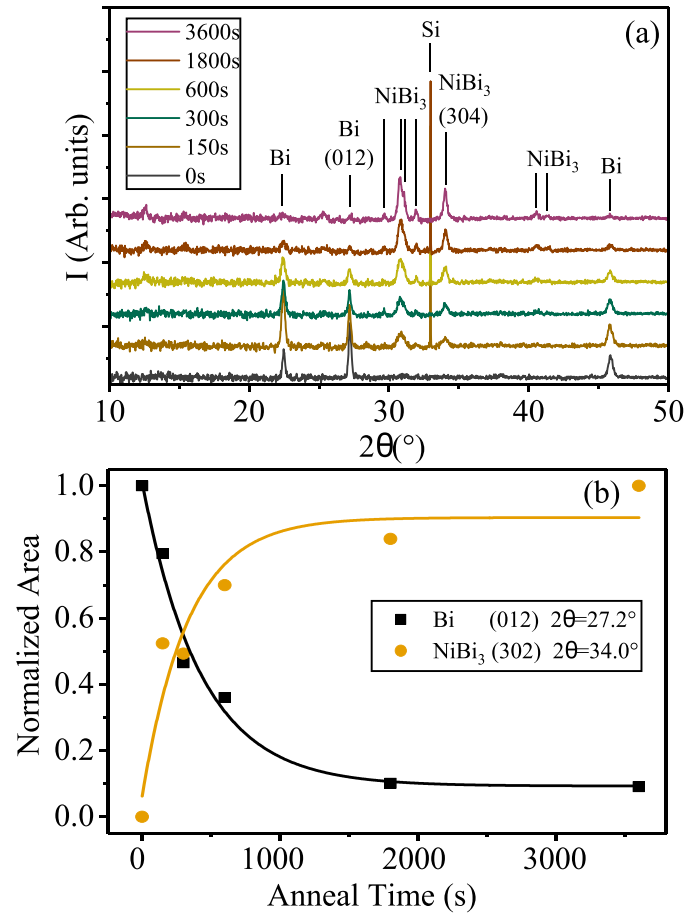

FIG. 4. X-ray diffraction characterization of $70^{\circ} \mathrm{C}$ annealed $\mathrm{Si} / \mathrm{SiO}(100 \mathrm{~nm}) / \mathrm{Bi}(50 \mathrm{~nm}) / \mathrm{Ni}(6 \mathrm{~nm}) / \mathrm{Ta}(5 \mathrm{~nm})$ samples. (a) Surfaced aligned XRD scans with annealing time. Peaks due to the $\mathrm{Si}$ substrate, $\mathrm{Bi}$ layer, and $\mathrm{NiBi}_{3}$ intermetallic are indexed. (b) The normalized area under the $\mathrm{Bi}(012)$ and $\mathrm{NiBi}_{3}$ (302) peaks to show evolution of the sample with annealing step. Lines are a guide to the eye.

two changes to the hysteresis loops are observed; First, the saturation moment/area of the sample reduces, and second, the coercive field increases. Both observations are consistent with the PNR modeling which shows that as the sample is annealed nonmagnetic $\mathrm{NiBi}_{3}$ intermetallic forms reducing the ferromagnetic Ni thickness.

It is possible to compare the measured magnetic moment returned from the SQUID measurements and PNR fitting (Fig. 2) by normalizing datasets to the areas of the samples. Collated moment/area with annealing time at $70^{\circ} \mathrm{C}$ for the samples are shown Fig. 3. As the sample is annealed we observe an exponential type decay of the sample moment/area from about $188 \mathrm{emu} / \mathrm{cm}^{2}$ in the as-grown state to $32 \mathrm{emu} / \mathrm{cm}^{2}$ after $3600 \mathrm{~s}$ annealing at $70^{\circ} \mathrm{C}$. The time constant of the decay is $530 \mathrm{~s}$. The extracted moment/area by the two techniques show close agreement in both trend and absolute value for annealed samples, we note that there is some disagreement in the magnetic moment of the as-grown state.

Figure 4(a) shows the results of XRD characterization. In the as-grown state, structural peaks due to the Bi layer and $\mathrm{Si}$ substrate are present in the sample. We do not expect $\mathrm{Ni}$ or Ta peaks to appear as these layers are too thin. After annealing, peaks appear in the XRD scan which correspond to the $\mathrm{NiBi}_{3}$ intermetallic and equally the Bi peak intensity drops, suggesting that the textured $\mathrm{Bi}$ layer is being replaced by a textured $\mathrm{NiBi}_{3}$ intermetallic. The timescale for these structural changes to occur to the sample is similar to the timescale where changes are observed in PNR and SQUID 

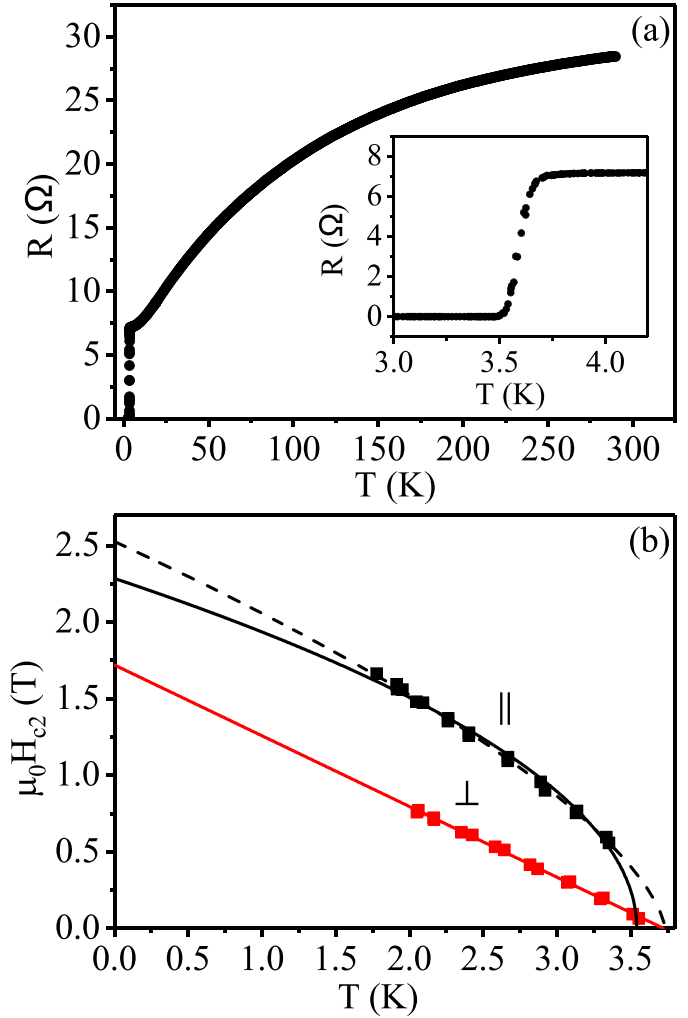

FIG. 5. Electrical transport characteristic of $\mathrm{Si} / \mathrm{SiO}(100 \mathrm{~nm}) / \mathrm{Bi}$ $(50 \mathrm{~nm}) / \mathrm{Ni}(6 \mathrm{~nm}) / \mathrm{Ta}(5 \mathrm{~nm})$ sample after $70^{\circ} \mathrm{C}$ annealing for 3600 s. (a) Resistance versus temperature with the superconducting transition at $3.8 \mathrm{~K}$ shown in the inset. (b) The out-of-plane (red) and in-plane (black) $H_{c 2}$ data, solid lines a model fit for a thin superconductor [Eqs. (1) and (2)]. The dashed line has the power of $\frac{1}{2}$ [Eq. (2)] as a free fitting parameter and the $T_{c}$ is fixed to the $T_{c}$ measured by the RvT. $\mu_{0} H_{c 2}^{\|}=2.28 \mathrm{~T}$ and $\mu_{0} H_{c 2}^{\perp}=1.72 \mathrm{~T}$, the power of the dash line is 0.659 .

measurements annealed at the same temperature. $\mathrm{The}^{\mathrm{NiBi}}{ }_{3}$ has a weakly preferred orientation toward (203).

\section{Superconducting properties}

Low-temperature four-point probe transport measurements of a $70^{\circ} \mathrm{C}$ fully annealed sample (3600 s) show a clear superconducting transition at $3.8 \mathrm{k}$ [Fig. 5(a)]; the critical magnetic field in-plane and out-of-plane was also measured [Fig. 5(b)]. The out-of-plane and in-plane $H_{c 2}$ temperature dependence can be fitted effectively by the following Ginzburg-Landau (GL) model for a thin superconductor:

$$
\begin{gathered}
\mu_{0} H_{c 2}^{\perp}=\frac{\Phi_{0}}{2 \pi \xi_{\mathrm{GL}}(0)^{2}}\left(1-\frac{T}{T_{c}}\right), \\
\mu_{0} H_{c 2}^{\|}=\frac{\Phi_{0} \sqrt{12}}{2 \pi \xi_{\mathrm{GL}}(0) d_{\mathrm{SC}}}\left(1-\frac{T}{T_{c}}\right)^{\frac{1}{2}},
\end{gathered}
$$

where $\Phi_{0}, \xi_{\mathrm{GL}}(0)$, and $d_{\mathrm{SC}}$ stands for flux quantum, in-plane coherence length and effective thickness of the superconductivity, respectively. Taking the values from both in-plane and out-of-plane $\mathrm{H}_{c 2}(0)$ fits the coherence length $\xi_{\mathrm{GL}}$ as $13.8 \mathrm{~nm}$ and the effective superconducting thickness $d_{\mathrm{SC}}$ as $36.2 \mathrm{~nm}$, similar to the thickness of the $\mathrm{NiBi}_{3}$ layer as obtained from the
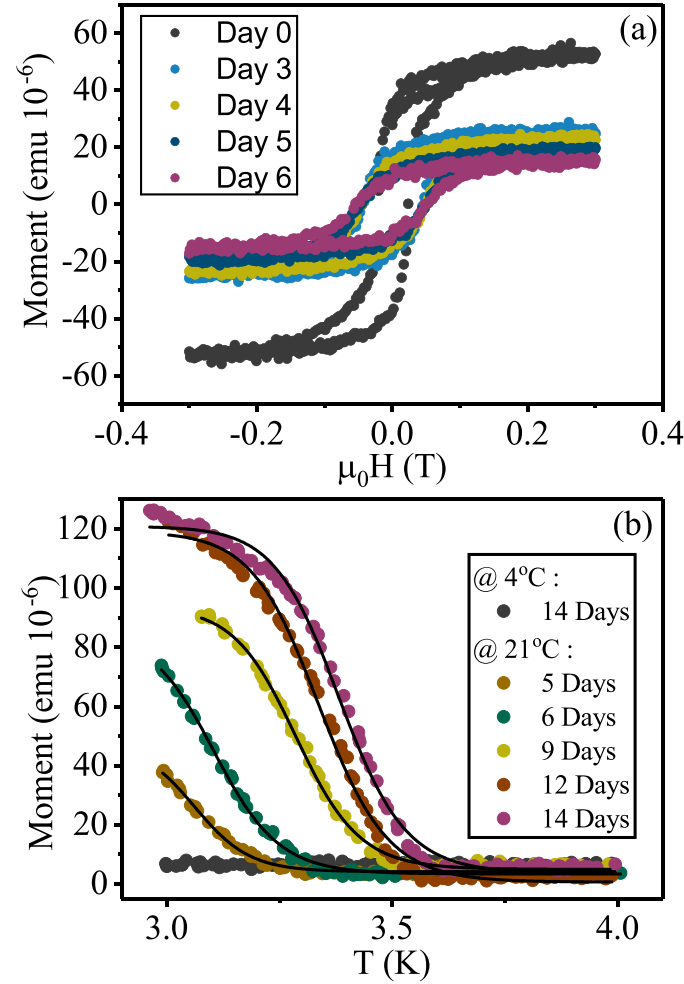

FIG. 6. (a) Sample of $\mathrm{Ta}(5) / \mathrm{Ni}(6) / \mathrm{Bi}(50) \mathrm{nm}$ stored at room temperature and hysteresis measured at $3 \mathrm{~K}$. (b) MvT of $\mathrm{Ta}(5) / \mathrm{Ni}(4) / \mathrm{Bi}(50) \mathrm{nm}$ samples at two different temperatures, black lines are logistic function fitting for which the $T_{c}$ is taken at $20 \%$ height.

PNR data. The GL-theory for thin superconductors assumes that the $d_{\mathrm{SC}}<\xi_{\mathrm{GL}}$ which is not the case here and is visible in Fig. 5(b) from the inadequate fitting for the in-plane $H_{c 2}$ data. Alternatively, making the power of the in-plane equation a free parameter (instead of a fixed $\frac{1}{2}$ ) returns a value of 0.659 and a more satisfactory fit to the experimental data. A returned power of 1 is expected for bulk behavior, suggesting this sample is in some intermediate state between bulk and thin superconductivity.

\section{E. Influence of annealing temperature}

Samples as-grown were not immediately observed to be superconducting, but after annealing or leaving at room temperature for several days the magnetization of the $\mathrm{Ni}$ layer was reduced although it is nonvanishing and an increased coercivity [Fig. 6(a)], after that a superconducting transition appeared and increased until stabilizing at $3.8 \mathrm{k}$ [Fig. 6(b)]. The thickness of the $\mathrm{NiBi}_{3}$ layer grows quickly as the sample is annealed once the layer is thick enough to support a superconducting transition there is a Meissner response that can be measured to deduce the $T_{c}$. By measuring the $T_{c}$ at several points along the annealing process a time constant for the onset can be fitted from a decaying exponential. As long as the sample is far from saturating its $T_{c}$ the time constant will be not be affect by small amounts of annealing that may have already taken place. The inverse time constant against annealing temperature can be described well by the 

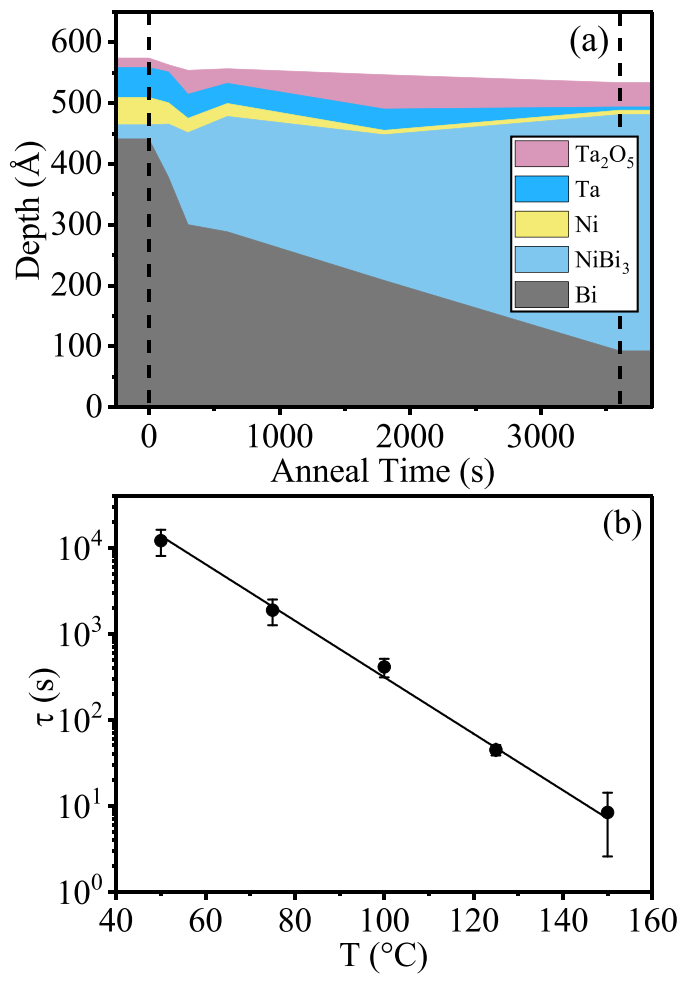

FIG. 7. (a) Fitted thickness extracted from PNR data for each annealing step, omitting the roughness at the interface for simplicity. The annealing at $70^{\circ} \mathrm{C}$ takes place between the dashed lines. (b) Arrhenius plot of $\mathrm{Bi} / \mathrm{Ni}$ bilayers annealed in the temperature range of $50^{\circ} \mathrm{C}-150^{\circ} \mathrm{C}$ with an activation energy of $(0.86 \pm 0.06) \mathrm{eV}$, time constants taken from the superconducting $T_{c}$ onset rate.

Arrhenius equation for a thermally activated reaction with an association activation energy of $(0.86 \pm 0.06) \mathrm{eV}$ [Fig. 7(b)]. The activation energy is lower than for similar systems of interface mixing and diffusion with typical activation energies of $\approx 1 \mathrm{eV}[25,26]$.

\section{DISCUSSION}

Figure 7(a) shows the fitted thickness for each annealing step as extracted from fitting to PNR. The $\mathrm{Ta}_{2} \mathrm{O}_{5}$ increases as expected for heating in air and has protected the lower layers from oxidation. The thickness of the $\mathrm{Bi}$ and $\mathrm{Ni}$ layers reduce as the $\mathrm{NiBi}_{3}$ layer becomes thicker. We also observe an overall reduction in film thickness as the $\mathrm{NiBi}_{3}$ alloy is denser than the individual $\mathrm{Bi}$ or Ni layers. To optimize the $T_{c} 50 \mathrm{~nm}$ of $\mathrm{Bi}$ and $6 \mathrm{~nm}$ of $\mathrm{Ni}$ are used (Fig. 1), the ratio maybe suggest that to obtain the highest $T_{c}$ annealed samples one should minimize the remaining $\mathrm{Ni}$ and maximize the thickness of the $\mathrm{NiBi}_{3}$.

Extrapolating from the Arrhenius plot at $21^{\circ} \mathrm{C}$ the time constant for the annealing rate will be 4 days which is comparable to the timescale measured at room temperature, the exponential has a doubling rate of $\approx 6^{\circ} \mathrm{C}$ such that if kept at $3^{\circ} \mathrm{C}$ the timescale extends to 12 days (Fig. 7). The low annealing temperatures can be understood from the low melting point of $\mathrm{Bi}$ of $544 \mathrm{~K}$ being about twice room temperature $(\approx 294 \mathrm{~K})$. It is not uncommon to anneal thin films to form alloys at $50 \%$ of the constituent layers melting points. The $\mathrm{Bi}$ atoms become mobile at higher temperatures diffusing across the interface and the same for $\mathrm{Ni}$ as it diffuses into the Bi layer forming a $\mathrm{NiBi}_{3}$ alloy.

When first measured in PNR, the as-grown sample has a thin layer at the interface with a density similar to $\mathrm{NiBi}_{3}$, although in the corresponding $\mathrm{XRD}$ the $\mathrm{NiBi}_{3}$ peaks are at the limit of signal to noise and so the volume fraction of ordered $\mathrm{NiBi}_{3}$ is very small. In comparison, in samples that have been intentionally annealed, the ratio for $\mathrm{NiBi}_{3}$ XRD peak areas and the fitted layer thickness suggest that $2 \mathrm{~nm}$ of $\mathrm{NiBi}_{3}$ should have a greater intensity of XRD peaks than is measured in the as-grown sample. This thin intermixed layer is not unexpected. The roughness of the Bi layer, implantation of $\mathrm{Ni}$ adatoms during growth and, probably most significantly, the time taken to get the samples from a room temperature vacuum chamber to the freezer all contribute to some intermixing of the interface.

Liu et al. perform pulsed laser deposition (PLD) of $\mathrm{Bi} / \mathrm{Ni}$ bilayers where they report the $\mathrm{NiBi}_{3}$ alloy forms during growth. Their interpretation is that PLD is a nonequilibrium process such that $\mathrm{Ni}$ atoms arrive at the $\mathrm{Bi}$ layer with enough energy to implant deep into the film. Ni implantation stops at the $\mathrm{SiO}_{2}$, where it accumulates forming a $\mathrm{NiBi}$ alloy and a $\mathrm{NiBi}_{3}$ layer further from the $\mathrm{SiO}_{2}$. We find that ordered $\mathrm{NiBi}_{3}$ does not exist in our samples at growth, but forms during annealing and is confined to the $\mathrm{Bi} / \mathrm{Ni}$ interface. We do not find evidence for ordered NiBi alloy in our films, however in the final annealed state Bi may have contaminated any remaining Ni layer in the structure. We conclude that during growth the Ni implantation depth is confined to near the top surface of the $\mathrm{Bi}$, as fitted by the $2 \mathrm{~nm}$ of $\mathrm{NiBi}_{3}$ in the as-grown state.

By measuring PNR below $T_{c}$ in an applied field, it is possible to observe Meissner screening under the right conditions (that the film thickness, roughness, and the superconducting penetration depth are balanced to allow for measurable screening to occur) [27,28]. It is also possible that exotic superconducting states can influence the magnetic response of a superconducting sample [29-31]. Here, we measure both the as-grown and fully annealed states of the sample at $3 \mathrm{~K}$ (below the superconducting transition for the annealed sample) to look for changes to magnetic response from the sample. No such changes are observed below $T_{c}$ in the PNR, most likely as the $\mathrm{NiBi}_{3}$ superconductor is too thin to observe Meissner screening.

Experimental results by other techniques suggest that superconductivity in bilayers of $\mathrm{Bi} / \mathrm{Ni}$ may be spin-triplet ( $p$ wave) in nature [10-16]. The symmetric spin-triplet states are found in only a handful of superconductors, where $\mathrm{Sr}_{2} \mathrm{RuO}_{4}$ is currently the best candidate [29]. In these materials, the antisymmetry requirements are satisfied by the condensates of these superconducting materials being spatially antisymmetric, that is, odd in angular momentum. It is also possible to generate spin-triplet states in proximity coupled thin films where $s$-wave pairing is retained by introducing a Berizinskii state with spontaneous breaking of time-reversal symmetry [32]. Our Bi/Ni samples contain all the necessary ingredients for such a state to occur: a source of $s$-wave superconductivity $\left(\mathrm{NiBi}_{3}\right)$, ferromagnetism $(\mathrm{Ni})$, and strong spin-orbit coupling (Bi) [33-36]. 


\section{CONCLUSIONS}

In this work samples of $\mathrm{Bi} / \mathrm{Ni}$ bilayers are observed initially to be nonsuperconducting until either left at room temperature for several days or a short period of heating as low as $+50^{\circ} \mathrm{C}$. The superconductivity is attributed to intermixing of the $\mathrm{Bi} / \mathrm{Ni}$ interface forming an alloy of $\mathrm{NiBi}_{3}$ identified by XRD Bragg peaks. PNR data is consistent with initially distinct $\mathrm{Bi} / \mathrm{Ni}$ layers with minimal $\mathrm{NiBi}_{3}$ in the as-grown states that when annealed at $70^{\circ} \mathrm{C}$ for $1 \mathrm{~h}$ the diffusion across the interface increases the $\mathrm{NiBi}_{3}$ thickness and reduces the pure $\mathrm{Bi} / \mathrm{Ni}$ layers.

From this it seems that to properly study clean and distinct interfaces that maintaining a low temperature for preparation and storage is important. Normal device fabrication recipes which use heating to bake resist or growth methods that allow the sample to heat up will fully anneal $\mathrm{Bi} / \mathrm{Ni}$ bilayers. Although the superconductivity origins from a known bulk superconductor the existence is still interesting as it is in close proximity to a ferromagnetic layer, a strong spin-orbit coupling $\mathrm{Bi}$ layer and also the likely proximity effect in the $\mathrm{Bi}$ layer.

The data associated with this paper are openly available from the University of Leeds and ISIS Neutron and Muon source data repositories [37].

\section{ACKNOWLEDGMENTS}

We thank the Science and Technology Facilities Council ISIS neutron and muon source for allocating beamtime (RB:1920455). The work was supported financially through the EPSRC Doctoral Training Partnership and Grant No. EP/M000923/1. This project has received funding from the European Union H2020 Marie Skłodowska-Curie Actions Grant Agreement No. 743791 (SUPERSPIN).
[1] V. L. Ginzburg, Zh. Eksp. Teor. Fiz. 31, 541 (1957) [Sov. Phys. JETP 4, 594 (1957)].

[2] O. Prakash, A. Kumar, A. Thamizhavel, and S. Ramakrishnan, Science 355, 52 (2017).

[3] V. I. Petrosyan, V. N. Molin, O. I. Vasin, P. A. Skripkina, S. I. Stenin, and E. G. Batyev, Zh. Eksp. Teor. Fiz. 66, 995 (1974) [Sov. Phys. JETP 39, 485 (1974)].

[4] W. Buckel and J. Wittig, Phys. Lett. 17, 187 (1965).

[5] B. Weitzel and H. Micklitz, Phys. Rev. Lett. 66, 385 (1991).

[6] Y. Li, E. Wang, X. Zhu, and H.-H. Wen, Phys. Rev. B 95, 024510 (2017).

[7] M. Tian, J. Wang, Q. Zhang, N. Kumar, T. E. Mallouk, and M. H. W. Chan, Nano Lett. 9, 3196 (2009).

[8] J. S. Moodera and R. Meservey, Phys. Rev. B 42, 179 (1990).

[9] P. LeClair, J. S. Moodera, J. Philip, and D. Heiman, Phys. Rev. Lett. 94, 037006 (2005).

[10] X.-X. Gong, H.-X. Zhou, P.-C. Xu, D. Yue, K. Zhu, X.-F. Jin, H. Tian, G.-J. Zhao, and T.-Y. Chen, Chin. Phys. Lett. 32, 067402 (2015).

[11] X. Gong, M. Kargarian, A. Stern, D. Yue, H. Zhou, X. Jin, V. M. Galitski, V. M. Yakovenko, and J. Xia, Sci. Adv. 3, e1602579 (2017).

[12] J. Wang, X. Gong, G. Yang, Z. Lyu, Y. Pang, G. Liu, Z. Ji, J. Fan, X. Jing, C. Yang, F. Qu, X. Jin, and L. Lu, Phys. Rev. B 96, 054519 (2017).

[13] H. Zhou and X. Jin, J. Magn. Magn. Mater. 458, 171 (2018).

[14] H. Zhou, X. Gong, and X. Jin, J. Magn. Magn. Mater. 422, 73 (2017).

[15] P. Chauhan, F. Mahmood, D. Yue, P.-C. Xu, X. Jin, and N. P. Armitage, Phys. Rev. Lett. 122, 017002 (2019).

[16] M. Tokuda, N. Kabeya, K. Iwashita, H. Taniguchi, T. Arakawa, D. Yue, X. Gong, X. Jin, K. Kobayashi, and Y. Niimi, Appl. Phys. Express 12, 53005 (2019).

[17] N. E. Alekseevskii, N. B. Brandt, and T. I. Kostina, Izv. Akad. Nauk SSSR, Ser. Fiz. 16, 233 (1952).
[18] V. Siva, K. Senapati, B. Satpati, S. Prusty, D. K. Avasthi, D. Kanjilal, and P. K. Sahoo, J. Appl. Phys. 117, 83902 (2015).

[19] B. Silva, R. F. Luccas, N. M. Nemes, J. Hanko, M. R. Osorio, P. Kulkarni, F. Mompean, M. García-Hernández, M. A. Ramos, S. Vieira, and H. Suderow, Phys. Rev. B 88, 184508 (2013).

[20] G. J. Zhao, X. X. Gong, P. C. Xu, B. C. Li, Z. Y. Huang, X. F. Jin, X. D. Zhu, and T. Y. Chen, Supercond. Sci. Technol. 31, 125005 (2018).

[21] L. Y. Liu, Y. T. Xing, I. L. C. Merino, H. Micklitz, D. F. Franceschini, E. Baggio-Saitovitch, D. C. Bell, and I. G. Solórzano, Phys. Rev. Mater. 2, 014601 (2018).

[22] M. Björck and G. Andersson, J. Appl. Cryst. 40, 1174 (2007).

[23] H. Zabel, K. Theis Brohl, M. Wolff, and B. Toperverg, IEEE Trans. Magn. 44, 1928 (2008).

[24] J. W. A. Robinson, S. Piano, G. Burnell, C. Bell, and M. G. Blamire, Phys. Rev. B 76, 094522 (2007).

[25] M.-A. Nicolet, Thin Solid Films 52, 415 (1978).

[26] H. J. Höfler, R. S. Averback, H. Hahn, and H. Gleiter, J. Appl. Phys. 74, 3832 (1993).

[27] H. Zhang, J. W. Lynn, C. F. Majkrzak, S. K. Satija, J. H. Kang, and X. D. Wu, Phys. Rev. B 52, 10395 (1995).

[28] A. J. Drew, M. W. Wisemayer, D. O. G. Heron, S. Lister, S. L. Lee, A. Potenza, C. H. Marrows, R. M. Dalgliesh, T. R. Charlton, and S. Langridge, Phys. Rev. B 80, 134510 (2009).

[29] G. M. Luke, Y. Fudamoto, K. M. Kojima, M. I. Larkin, J. Merrin, B. Nachumi, Y. J. Uemura, Y. Maeno, Z. Q. Mao, Y. Mori, H. Nakamura, and M. Sigrist, Nature 394, 558 (1998).

[30] M. G. Flokstra, N. Satchell, J. Kim, G. Burnell, P. J. Curran, S. J. Bending, J. F. K. Cooper, C. J. Kinane, S. Langridge, A. Isidori, N. Pugach, M. Eschrig, H. Luetkens, A. Suter, T. Prokscha, and S. L. Lee, Nat. Phys. 12, 57 (2016).

[31] M. G. Flokstra, R. Stewart, N. Satchell, G. Burnell, H. Luetkens, T. Prokscha, A. Suter, E. Morenzoni, S. Langridge, and S. L. Lee, Appl. Phys. Lett. 115, 072602 (2019). 
[32] F. S. Bergeret, A. F. Volkov, and K. B. Efetov, Phys. Rev. Lett. 86, 4096 (2001).

[33] F. S. Bergeret and I. V. Tokatly, Phys. Rev. B 89, 134517 (2014).

[34] K.-R. Jeon, C. Ciccarelli, A. J. Ferguson, H. Kurebayashi, L. F. Cohen, X. Montiel, M. Eschrig, J. W. A. Robinson, and M. G. Blamire, Nat. Mater. 17, 499 (2018).
[35] N. Satchell and N. O. Birge, Phys. Rev. B 97, 214509 (2018).

[36] N. Satchell, R. Loloee, and N. O. Birge, Phys. Rev. B 99, 174519 (2019).

[37] M. Vaughan et al., University of Leeds Data Repository: https://doi.org/10.5518/740; ISIS Neutron and Muon Source Data Repository: doi:10.5286/ISIS.E.RB1920455 (2019). 